\title{
Elliptic Biorthogonal Polynomials Connected with Hermite's Continued Fraction ${ }^{\star}$
}

Luc VINET ${ }^{\dagger}$ and Alexei ZHEDANOV

† Université de Montréal, PO Box 6128, Station Centre-ville, Montréal QC H3C 3J7, Canada E-mail: luc.vinet@umontreal.ca

$\ddagger$ Donetsk Institute for Physics and Technology, Donetsk 83114, Ukraine

E-mail: zhedanov@yahoo.com

Received October 07, 2006, in final form December 12, 2006; Published online January 04, 2007

Original article is available at http://www.emis.de/journals/SIGMA/2007/003/

\begin{abstract}
We study a family of the Laurent biorthogonal polynomials arising from the Hermite continued fraction for a ratio of two complete elliptic integrals. Recurrence coefficients, explicit expression and the weight function for these polynomials are obtained. We construct also a new explicit example of the Szegö polynomials orthogonal on the unit circle. Relations with associated Legendre polynomials are considered.
\end{abstract}

Key words: Laurent biorthogonal polynomials; associated Legendre polynomials; elliptic integrals

2000 Mathematics Subject Classification: 33C45; 42C05

To the memory of Vadim B. Kuznetsov

Vadim Kuznetsov spent a number of years at the Centre de Recherches Mathématiques of the Université de Montréal in the mid-90s. This is when we had the privilege to have him as a colleague and the chance to appreciate his scientif ic and human qualities. His smile, great spirit, penetrating insights and outstanding scientif ic contributions will always be with us.

\section{Introduction}

We start with well known identity (see e.g. [24])

$$
\frac{d^{2} \operatorname{sn}^{n} u}{d u^{2}}=n(n-1) \operatorname{sn}^{n-2} u-n^{2}\left(1+k^{2}\right) \operatorname{sn}^{n} u+n(n+1) k^{2} \operatorname{sn}^{n+2} u
$$

for the Jacobi elliptic function sn $u$ depending on an argument $u$ and a modulus $k$. This (and similar 11 formulas obtained if one replaces $s n$ with other elliptic functions) identity goes back to Jacobi and usually is exploited in order to establish recurrence relations for elliptic integrals.

Indeed, introduce the following elliptic integrals

$$
J_{n}(x ; k)=\int_{0}^{v} k^{2 n} \operatorname{sn}^{2 n} u d u=\int_{0}^{x} \frac{k^{2 n} t^{2 n} d t}{\sqrt{\left(1-t^{2}\right)\left(1-k^{2} t^{2}\right)}},
$$

where $\operatorname{sn} u=t$ and $x=\operatorname{sn} v$. Then from (1.1) we obtain

$$
(2 n-1) J_{n}(x ; k)-(2 n-2)\left(k^{2}+1\right) J_{n-1}(x ; k)+k^{2}(2 n-3) J_{n-2}(x ; k)
$$

${ }^{\star}$ This paper is a contribution to the Vadim Kuznetsov Memorial Issue "Integrable Systems and Related Topics". The full collection is available at http://www.emis.de/journals/SIGMA/kuznetsov.html 


$$
=k^{2 n-2} x^{2 n-3} \sqrt{\left(1-x^{2}\right)\left(1-k^{2} x^{2}\right)} .
$$

From this formula we can express $J_{n+1}(x ; k)$ in the form [12]

$$
J_{n+1}(x ; k)=Q(x ; n) \sqrt{\left(1-x^{2}\right)\left(1-k^{2} x^{2}\right)}+A_{n} J_{1}(x ; k)-B_{n} J_{0}(x ; k),
$$

where $Q(x ; n)$ is a polynomial in $x$ (depending on $n$ ) and the coefficients $A_{n}, B_{n}$ satisfy the same recurrence relations as $J_{n+1}(1 ; k)$, i.e.

$$
\begin{aligned}
& (2 n+1) A_{n}-2 n\left(1+k^{2}\right) A_{n-1}+(2 n-1) k^{2} A_{n-2}=0 \\
& (2 n+1) B_{n}-2 n\left(1+k^{2}\right) B_{n-1}+(2 n-1) k^{2} B_{n-2}=0
\end{aligned}
$$

with obvious initial conditions

$$
A_{-1}=0, \quad B_{-1}=-1, \quad A_{0}=1, \quad B_{0}=0 .
$$

Note that from recurrence relations (1.5) and initial conditions (1.6) it follows that $A_{n}(k)$ is a polynomial in $k^{2}$ of degree $n$ and $B_{n}(k)$ is a polynomial in $k^{2}$ of degree $n$ but having common factor $k^{2}$, i.e. $B_{n}=k^{2} V_{n-1}\left(k^{2}\right)$, where $V_{n-1}(z)$ is a polynomial of degree $n-1$ in $z$ for any $n=1,2, \ldots$.

There is an elementary Wronskian-type identity following directly from (1.5) [12]:

$$
B_{n} A_{n-1}-A_{n} B_{n-1}=\frac{k^{2 n}}{2 n+1} .
$$

Formula (1.4) allows to reduce calculation of any (incomplete) elliptic integrals of the form

$$
\int_{0}^{x} \frac{P(t) d t}{\sqrt{\left(1-t^{2}\right)\left(1-k^{2} t^{2}\right)}}
$$

(where $P(t)$ is a polynomial) to standard elliptic integrals of the first and second kind $J_{0}(x ; k)$ and $J_{1}(x ; k)$. This result is well known since Jacobi.

In what follows we will denote $J_{0}(1 ; k)=K(k)$ and $J_{1}(1 ; k)=J(k)$. Note that $K(k)$ is the standard complete elliptic integral of the first kind [24] and

$$
J(k)=K(k)-E(k)=-k \frac{d E}{d k},
$$

where

$$
E(k)=\int_{0}^{1}\left(\frac{1-k^{2} x^{2}}{1-x^{2}}\right)^{1 / 2} d x
$$

is the complete elliptic integral of the second kind.

We note also an important relation with hypergeometric functions [24]:

$$
\begin{aligned}
& K(k)=\frac{\pi}{2}{ }_{2} F_{1}\left(1 / 2,1 / 2 ; 1 ; k^{2}\right), \quad E(k)=\frac{\pi}{2}{ }_{2} F_{1}\left(-1 / 2,1 / 2 ; 1 ; k^{2}\right), \\
& J(k)=\frac{\pi k^{2}}{4}{ }_{2} F_{1}\left(3 / 2,1 / 2 ; 2 ; k^{2}\right) .
\end{aligned}
$$

Hermite in his famous "Cours d'analyse" [12] (see also [10, 11]) derived a continued fraction connected with a ratio of two complete elliptic integrals:

$$
\frac{J(k)}{K(k)}=\frac{k^{2}}{2\left(1+k^{2}\right)-\frac{9 k^{2}}{4\left(1+k^{2}\right)-\frac{25 k^{2}}{6\left(1+k^{2}\right)-\cdots}}},
$$


where

$$
K(k)=\int_{0}^{1} \frac{d x}{\sqrt{\left(1-x^{2}\right)\left(1-k^{2} x^{2}\right)}}, \quad J(k)=\int_{0}^{1} \frac{k^{2} x^{2} d x}{\sqrt{\left(1-x^{2}\right)\left(1-k^{2} x^{2}\right)}} .
$$

The Hermite continued fraction (1.9) follows directly from (1.3). Note that the continued fraction (1.9) belongs to the class of the so-called T-continued fractions (with respect to the variable $k^{2}$ ) introduced and studied by Thron (see [14] for details). Thus perhaps Hermite was the first to introduce an explicit example of the $T$-continued fraction. In what follows we will see that this example gives rise to a class of polynomials which are biorthogonal on the unit circle.

Rewrite relation (1.4) for $x=1$ in the form

$$
J(k) / K(k)-B_{n} / A_{n}=\frac{J_{n+1}(k)}{K(k) A_{n}}
$$

from which Hermite concluded that the rational function $B_{n} / A_{n}$ is an approximate expression for the ratio $J(k) / K(k)$ to within terms of degree $n+1$ in $k^{2}$.

Hermite also noted that the coefficients $A_{n}, B_{n}$ appeared as power coefficients in the following Taylor expansions:

$$
\begin{aligned}
& \frac{J_{0}(x)}{\sqrt{\left(1-x^{2}\right)\left(1-k^{2} x^{2}\right)}}=A_{0} x+A_{1} x^{3}+\cdots+A_{n} x^{2 n+1}+\cdots, \\
& \frac{J_{1}(x)}{\sqrt{\left(1-x^{2}\right)\left(1-k^{2} x^{2}\right)}}=B_{0} x+B_{1} x^{3}+\cdots+B_{n} x^{2 n+1}+\cdots .
\end{aligned}
$$

Thus formulas (1.11) can be considered as generating functions for $A_{n}, B_{n}$.

\section{Laurent biorthogonal polynomials}

Introduce the new variable $z=k^{2}$ and define the polynomials $P_{n}(z)=A_{n} / \xi_{n}$ of degree $n$ in $z$, where

$$
\frac{\xi_{n}}{\xi_{n+1}}=\frac{2 n+3}{2 n+2}, \quad \xi_{0}=1 .
$$

We have

$$
\xi_{n}=\frac{n !}{(3 / 2)_{n}},
$$

where $(a)_{n}=a(a+1) \cdots(a+n-1)$ is the standard Pochhammer symbol (shifted factorial).

Then it is seen that $P_{n}(z)=z^{n}+O\left(z^{n-1}\right)$, i.e. $P_{n}(z)$ are monic polynomials. Moreover from (1.5) it follows that $P_{n}(z)$ satisfy the 3 -term recurrence relation

$$
P_{n+1}(z)+d_{n} P_{n}(z)=z\left(P_{n}(z)+b_{n} P_{n-1}(z)\right)
$$

where

$$
d_{n}=-1, \quad n=0,1, \ldots, \quad b_{n}=-\frac{(n+1 / 2)^{2}}{n(n+1)}, \quad n=1,2, \ldots
$$

with initial conditions

$$
P_{-1}(z)=0, \quad P_{0}(z)=1
$$


Define also the polynomials

$$
P_{n-1}^{(1)}(z)=2 B_{n} /\left(z \xi_{n}\right) .
$$

It is then easily verified that the polynomials $P_{n}^{(1)}(z)$ are again $n$-th degree monic polynomials in $z$ satisfying the recurrence relation

$$
P_{n+1}^{(1)}(z)+d_{n+1} P_{n}^{(1)}(z)=z\left(P_{n}^{(1)}(z)+b_{n+1} P_{n-1}^{(1)}(z)\right) .
$$

The polynomials $P_{n}(z)$ are Laurent biorthogonal polynomials (LBP) [7] and the polynomials $P_{n}^{(1)}(z)$ are the corresponding associated LBP. The recurrence coefficients $b_{n}, d_{n}$ completely characterize LBP. The nondegeneracy condition $b_{n} d_{n} \neq 0, n=1,2, \ldots[7,27]$ obviously holds in our case.

It is well known that LBP possess the biorthogonality property [7]. This means that there exists a family of other LBP $\hat{P}_{n}(z)$ and a linear functional $\sigma$ such that

$$
\left\langle\sigma, P_{n}(z) \hat{P}_{m}(1 / z)\right\rangle=h_{m} \delta_{n m},
$$

where the normalization constants $h_{n}$ are expressed as [7]

$$
h_{n}=\prod_{k=1}^{n} \frac{b_{k}}{d_{k}} .
$$

The linear functional $\sigma$ is defined on the space of all monomials $z^{s}$ with both positive and negative values of $s$ :

$$
c_{s}=\left\langle\sigma, z^{s}\right\rangle, \quad s=0, \pm 1, \pm 2, \ldots,
$$

where $c_{s}$ is a sequence of moments (this sequence is infinite in both directions).

The biorthogonality condition (2.6) is equivalent (under the nondegeneracy condition $h_{n} \neq 0$ ) to the orthogonality relations

$$
\left\langle\sigma, P_{n}(z) z^{-j}\right\rangle=0, \quad j=0,1, \ldots, n-1 .
$$

Note that in our case from (2.2) and (2.3) it follows that

$$
P_{n}(0)=1, \quad n=0,1,2, \ldots
$$

Introduce the reciprocal LBP by the formula [23]

$$
P_{n}^{*}(z)=\frac{z^{n} P_{n}(1 / z)}{P_{n}(0)} .
$$

It appears that $P_{n}^{*}(z)$ are again LBP with the recurrence coefficients [23]

$$
b_{n}^{*}=\frac{b_{n}}{d_{n} d_{n+1}}, \quad d_{n}^{*}=\frac{1}{d_{n}} .
$$

The moments $c_{n}^{*}$ of the reciprocal polynomials are expressed as [23]

$$
c_{n}^{*}=\frac{c_{1-n}}{c_{1}} .
$$

In our case (2.3) we have $b_{n}^{*}=b_{n}, d_{n}^{*}=d_{n}$. Hence the reciprocal polynomials coincide with the initial ones:

$$
z^{n} P_{n}(1 / z)=P_{n}(z) .
$$

Moreover in our case $c_{1}=d_{0}=-1$ and thus $c_{n}^{*}=-c_{1-n}$. 
The polynomials $\hat{P}_{n}(z)$ are the biorthogonal partners with respect to the polynomials $P_{n}(z)$. Their moment sequence $\hat{c}_{n}$ is obtained from the initial moment sequence by reflection:

$$
\hat{c}_{n}=c_{-n}, \quad n=0, \pm 1, \pm 2, \ldots
$$

Note that under the assumption $c_{0}=1$ (standard normalization condition) we have $\hat{c}_{0}=1$ as well.

For the biorthogonal partners there is an explicit expression $[7,23]$

$$
\hat{P}_{n}(z)=\frac{z^{n} P_{n+1}(1 / z)-z^{n-1} P_{n}(1 / z)}{P_{n+1}(0)} .
$$

In our case (taking into account properties (2.9) and (2.13)) it is seen that

$$
\hat{P}_{n}(z)=\frac{P_{n+1}(z)-P_{n}(z)}{z} .
$$

Formula (2.16) admits another interpretation if one introduces the Christoffel transform (CT) of LBP. Recall $[27,23,22]$ that the CT for LBP is defined as

$$
P_{n}^{(C)}(z)=\frac{P_{n+1}(z)-U_{n} P_{n}(z)}{z-\mu}, \quad n=0,1, \ldots, \quad U_{n}=\frac{P_{n+1}(\mu)}{P_{n}(\mu)},
$$

where $\mu$ is an arbitrary parameter such that $P_{n}(\mu) \neq 0, n=1,2, \ldots$ The polynomials $P_{n}^{(C)}(z)$ are again monic LBP with the transformed recurrence coefficients

$$
b_{n}^{(C)}=b_{n} \frac{b_{n+1}+U_{n}}{b_{n}+U_{n-1}}, \quad d_{n}^{(C)}=d_{n} \frac{d_{n+1}+U_{n+1}}{d_{n}+U_{n}} .
$$

The moments $c_{n}^{(C)}$ corresponding to CT are expressed as [27]

$$
c_{n}^{(C)}=\frac{c_{n+1}-\mu c_{n}}{c_{1}-\mu}
$$

There is a special case of the CT when $\mu=0$. In this case, we have

$$
P_{n}^{(C)}(z)=\frac{P_{n+1}(z)+d_{n} P_{n}(z)}{z} .
$$

For the recurrence coefficients in this case we have [27]

$$
\begin{aligned}
& \tilde{b}_{n}=b_{n} \frac{b_{n+1}-d_{n}}{b_{n}-d_{n-1}}, \quad n=1,2, \ldots, \\
& \tilde{d}_{0}=d_{0}-b_{1}, \quad \tilde{d}_{n}=d_{n-1} \frac{b_{n+1}-d_{n}}{b_{n}-d_{n-1}}, \quad n=1,2, \ldots
\end{aligned}
$$

Note that there is some "irregularity" in the expression for $\tilde{d}_{n}$ in $(2.21)$ for $n=0$ and $n=1,2, \ldots$. This irregularity can be avoided if one formally puts $b_{0}=0$. However in our case $b_{0} \neq 0$. Hence we will indeed have such an irregularity in the analytic dependence of the coefficients $\tilde{d}_{n}$ in $n$. Namely, we have the explicit expressions

$$
\begin{aligned}
& \tilde{b}_{n}=-\frac{(n+1 / 2)^{2}}{(n+1)(n+2)}, \quad n=1,2, \ldots, \\
& \tilde{d}_{0}=1 / 8, \quad \tilde{d}_{n}=-\frac{n}{n+2}, \quad n=1,2, \ldots
\end{aligned}
$$


The corresponding moments are transformed simply as a "shift"

$$
c_{n}^{(C)}=\frac{c_{n+1}}{c_{1}} .
$$

Comparing (2.20) with (2.16) we see that in our case the biorthogonal partners $\hat{P}_{n}(z)$ coincide with the CT LBP $P_{n}^{(C)}$ for $\mu=0$. Taking into account that in our case $c_{1}=-1$ we obtain that the "negative" moments are expressed as

$$
c_{-n}=-c_{n+1}, \quad n=1,2, \ldots
$$

The LBP are connected with the two-point Padé approximation problem [7]. Given the moments $c_{n}, n=0, \pm 1, \pm 2, \ldots$, consider two formal power series

$$
F_{+}(z)=\sum_{k=1}^{\infty} c_{k} z^{-k}, \quad F_{-}(z)=\sum_{k=0}^{\infty} c_{-k} z^{k} .
$$

Then we have $[7,27]$

$$
\frac{P_{n-1}^{(1)}(z)}{P_{n}(z)}=\frac{F_{+}(z)}{c_{1}}+O\left(z^{-n-1}\right), \quad \frac{P_{n-1}^{(1)}(z)}{P_{n}(z)}=-\frac{F_{-}(z)}{c_{1}}+O\left(z^{n}\right) .
$$

It is convenient to introduce the formal Laurent series

$$
F(z)=\frac{F_{+}(z)-F_{-}(z)}{c_{1}} .
$$

Then we have

$$
F(z)-\frac{P_{n-1}^{(1)}(z)}{P_{n}(z)}= \begin{cases}O\left(z^{-n-1}\right), & z \rightarrow \infty \\ O\left(z^{n}\right), & z \rightarrow 0\end{cases}
$$

i.e. LBP $P_{n}(z), P_{n-1}^{(1)}(z)$ solve the problem of two-point Padé approximation (at $\left.z=0, \infty\right)$. Note that the Hermite formula (1.10) describes "one half" of this two-point Padé approximation problem.

So it is reasonable to refer to the polynomials $P_{n}(z)$ as the Hermite elliptic Laurent biorthogonal polynomials.

\section{The weight function and biorthogonality}

Consider the functions

$$
F_{-}(z)=-\frac{2 J(k)}{k^{2} K(k)}, \quad F_{+}(z)=-\frac{2 J(1 / k)}{K(1 / k)},
$$

where $z=k^{2}$. It is assumed that the function $F_{-}(z)$ is defined near $z=0$ while the function $F_{+}(z)$ is defined near $z=\infty$. Note that formally

$$
F_{+}(z)=\frac{F_{-}(1 / z)}{z}
$$

From the considerations of the previous section it is easily verified that formula (2.28) holds for the LBP defined by the recurrence coefficients (2.3). It is seen also that if one introduces the function (cf. [9])

$$
w(z)=\frac{F_{+}(z)-F_{-}(z)}{2 \pi i z}
$$


then

$$
\int_{C} z^{-k} w(z) d z=c_{k}, \quad k=0, \pm 1, \pm 2, \ldots
$$

where the integration contour $C$ is the unit circle. Thus the biorthogonality property (2.6) can be presented in the form

$$
\int_{C} P_{n}(z) \hat{P}_{m}(1 / z) w(z) d z=h_{n m} \delta_{n m} .
$$

Now we calculate the weight function $w(z)$ in a more explicit form. We have

$$
w(z)=\frac{1}{\pi i k^{2}}\left(-\frac{J(k)}{k^{2} K(k)}+\frac{J(1 / k)}{K(1 / k)}\right) .
$$

Using the relation $J(k)=K(k)-E(k)$ and the formulas for the complete elliptic integrals with inverse modulus (as usual $K^{\prime}(k)=K\left(k^{\prime}\right), E^{\prime}(k)=E\left(k^{\prime}\right), k^{\prime 2}=1-k^{2}$ ):

$$
K(1 / k)=k\left(K(k)+i K^{\prime}(k)\right), \quad E(1 / k)=\frac{E(k)-i E^{\prime}(k)-k^{\prime 2} K(k)+i k^{2} K^{\prime}(k)}{k}
$$

we arrive at the formula

$$
w(z)=\frac{-K(k) K^{\prime}(k)+E(k) K^{\prime}(k)+K(k) E^{\prime}(k)}{k^{4} K(k) K(1 / k)} .
$$

The latter expression can be further simplified using the Legendre relation [24]

$$
-K(k) K^{\prime}(k)+E(k) K^{\prime}(k)+K(k) E^{\prime}(k)=\pi / 2
$$

to give

$$
w(z)=\frac{1}{2 z^{3 / 2}} \frac{1}{K(k) K(1 / k)} .
$$

Now we introduce variable $\theta$ on the unit circle such that $k=z^{1 / 2}=e^{i \theta / 2}$. The biorthogonality relation can then be written as

$$
\int_{0}^{2 \pi} P_{n}\left(e^{i \theta}\right) \hat{P}_{m}\left(e^{-i \theta}\right) \rho(\theta) d \theta=h_{n} \delta_{n m}
$$

where the weight function is

$$
\rho(\theta)=\frac{i}{2 e^{i \theta / 2}\left|K\left(e^{i \theta / 2}\right)\right|^{2}} .
$$

\section{Generating function and explicit expression}

From (1.11) we obtain the generating function for the corresponding LBP

$$
\Phi(x, z)=\frac{F(x ; z)}{\sqrt{\left(1-x^{2}\right)\left(1-z x^{2}\right)}}=\sum_{n=0}^{\infty} \xi_{n} P_{n}(z),
$$

where $\xi_{n}$ is given by (2.1) and

$$
F(x ; z)=\int_{0}^{x} \frac{d t}{\sqrt{\left(1-t^{2}\right)\left(1-z t^{2}\right)}}
$$

is the standard (incomplete) elliptic integral of the first kind. 
In order to find the explicit expression for the polynomials $P_{n}(z)$ from (4.1), we note that if

$$
\frac{1}{\sqrt{\left(1-x^{2}\right)\left(1-z x^{2}\right)}}=\sum_{n=0}^{\infty} \beta_{n} x^{2 n}
$$

then, obviously,

$$
F(x ; z)=\sum_{n=0}^{\infty} \beta_{n} x^{2 n+1} /(2 n+1)
$$

and hence $\Phi(x, z)=\sum_{n=0}^{\infty} A_{n}(z) x^{2 n+1}$ where

$$
A_{n}(z)=\sum_{s=0}^{n} \frac{\beta_{s} \beta_{n-s}}{2 s+1} .
$$

Formula (4.3) gives an explicit expression for the polynomials $A_{n}(z)$ and hence for the LBP $P_{n}(z)$ if the coefficients $\beta_{n}$ are known. But it is easy to verify (using e.g. the binomial theorem) that

$$
\beta_{n}=\frac{(1 / 2)_{n}}{n !}{ }_{2} F_{1}(-n, 1 / 2 ; 1 / 2-n ; z) .
$$

We thus have

$$
\begin{aligned}
A_{n}(z)= & \sum_{m=0}^{n} \frac{(1 / 2)_{n}(1 / 2)_{m}(-n)_{m}}{(2 m+1) n ! m !(1 / 2-n)_{m}} \\
& \times{ }_{2} F_{1}(-m, 1 / 2 ; 1 / 2-m ; z)_{2} F_{1}(m-n, 1 / 2 ; 1 / 2+m-n ; z) .
\end{aligned}
$$

Another explicit expression is obtained if one notices that

$$
\frac{1}{\sqrt{\left(1-x^{2}\right)\left(1-k^{2} x^{2}\right)}}=\sum_{n=0}^{\infty} k^{n} x^{2 n} Y_{n}\left(\frac{k+k^{-1}}{2}\right),
$$

where $Y_{n}(z)$ are the ordinary Legendre polynomials [15]:

$$
Y_{n}(t)={ }_{2} F_{1}\left(-n, n+1 ; 1 ; \frac{1-t}{2}\right) .
$$

We thus have the rather simple expression

$$
A_{n}(z)=k^{n} \sum_{s=0}^{n} \frac{Y_{s}(q) Y_{n-s}(q)}{2 s+1}
$$

where $q=\left(k+k^{-1}\right) / 2$ (recall that $\left.k^{2}=z\right)$.

For $z=1$ we have $\beta_{n}=1$ for all $n$ in (4.2). Hence we have from (4.3)

$$
A_{n}(1)=G_{n},
$$

where we denote

$$
G_{n}=\sum_{s=0}^{n} \frac{1}{2 s+1}
$$


- the finite sum of inverse odd numbers. $G_{n}$ can be obviously expressed in terms of the Euler "harmonic numbers" defined as

$$
H_{n}=\sum_{k=1}^{n} 1 / k .
$$

We have clearly

$$
G_{n}=H_{2 n+1}-H_{n} / 2 .
$$

Consider the recurrence relation of the type (1.5)

$$
(2 n+1) \psi_{n}-2 n(1+z) \psi_{n-1}+(2 n-1) z \psi_{n-2}=0 .
$$

For $z=1$ we see that $\psi_{n}=G_{n}$ is a solution of this equation. The second independent solution for $z=1$ is trivial - it is a constant: $\psi_{n}=$ const. This means that the general solution of the equation (4.10) for $z=1$ can be presented in the form

$$
\psi_{n}=\alpha+\beta G_{n}
$$

with arbitrary constants $\alpha, \beta$. In particular, for $B_{n}(1)$ we can write

$$
B_{n}(1)=G_{n}-1
$$

\section{Polynomials orthogonal on the unit circle}

Consider the Christoffel transform (2.17) of our polynomials with $\mu=1$. We have

$$
U_{n}=\frac{P_{n+1}(1)}{P_{n}(1)}=\frac{\xi_{n} A_{n+1}(1)}{\xi_{n+1} A_{n}(1)}=\frac{(n+3 / 2) G_{n+1}}{(n+1) G_{n}} .
$$

For the corresponding transformed LBP, we have the expression

$$
\tilde{P}_{n}(z)=\frac{P_{n+1}(z)-U_{n} P_{n}(z)}{z-1} .
$$

The moments are calculated by (2.19):

$$
\tilde{c}_{n}=\frac{c_{n+1}-c_{n}}{c_{1}-1}=\frac{c_{n}-c_{n+1}}{2} .
$$

Using property $(2.24)$ we see that $\tilde{c}_{-n}=\tilde{c}_{n}$, i.e. the moments $\tilde{c}_{n}$ are symmetric with respect to

reflection. In turn, this is equivalent to the statement that the corresponding polynomials $\tilde{P}_{n}(z)$ are the Szegö polynomials which are orthogonal on the unit circle $[21,5]$ :

$$
\int_{0}^{2 \pi} \tilde{P}_{n}\left(e^{i \theta}\right) \tilde{P}_{m}\left(e^{-i \theta}\right) \tilde{\rho}(\theta) d \theta=h_{n} \delta_{n m}
$$

where

$$
\tilde{\rho}(\theta)=\frac{\rho(\theta)}{c_{1}-1}\left(e^{i \theta}-1\right)
$$

In our case we have explicitly (see (3.9))

$$
\tilde{\rho}(\theta)=\frac{\sin (\theta / 2)}{2\left|K\left(e^{i \theta / 2}\right)\right|^{2}} .
$$


It is well known that polynomials orthogonal on the unit circle are defined by the recurrence relation [5]

$$
P_{n+1}(z)=z P_{n}(z)-a_{n} P_{n}^{*}(z), \quad n=0,1, \ldots,
$$

where $P_{n}^{*}(z)=z^{n} \bar{P}_{n}(1 / z)$ (bar means complex conjugate). In our case all moments $\tilde{c}_{n}$ are real, hence $\tilde{P}_{n}^{*}(z)=z^{n} \tilde{P}_{n}(1 / z)$. The parameters $a_{n}$ are called the reflection parameters. They play a crucial role in the theory of Szegö polynomials on the unit circle. We have

$$
a_{n}=-\tilde{P}_{n+1}(0) \text {. }
$$

It is well known [5] that if the reflection parameters are real and satisfy the condition $\left|a_{n}\right|<1$ for all $n=0,1, \ldots$ then the positive weight function $\rho(\theta)>0$ always exists. Moreover in this case the weight function is symmetric on the unit circle: $\rho(2 \pi-\theta)=\rho(\theta)$.

In our case we have

$$
a_{n}=-\tilde{P}_{n+1}(0)=-U_{n+1} P_{n+1}(0)+P_{n+2}(0)=1-U_{n+1},
$$

where we used the property $P_{n}(0)=1$.

It is easily seen that

$$
a_{n}=-\frac{1}{2(n+2)}-\frac{1}{2(n+2) G(n+1)}, \quad n=0,1, \ldots
$$

In (5.10) both terms are negative and less then $1 / 2$ in absolute value. Hence $-1<a_{n}<0$ for all $n=0,1, \ldots$ and the function $\tilde{\rho}(\theta)$ is positive as is seen from (5.6).

We thus have a (presumably) new example of the Szegö polynomials orthogonal on the unit circle for which both weight function and recurrence coefficients are known explicitly.

Following [4] and [26], to any polynomials $\tilde{P}_{n}(z)$ orthogonal on the unit circle with the property $-1<a_{n}<1$ one can associate symmetric monic polynomials $S_{n}(x)=x^{n}+O\left(x^{n-1}\right)$ orthogonal on an interval of the real axis. Explicitly

$$
S_{n}(x)=\frac{z^{-n / 2}\left(\tilde{P}_{n}(z)+\tilde{P}_{n}^{*}(z)\right)}{1-a_{n-1}},
$$

where $x=z^{1 / 2}+z^{-1 / 2}$ (it is assumed that one chooses one branch of the function $z^{1 / 2}$ such that for $z=r e^{i \theta}$ we have $\left.z^{1 / 2}=r^{1 / 2} e^{i \theta / 2},-\pi<\theta<\pi\right)$. The polynomials $S_{n}(x)$ satisfy the three-term recurrence relation

$$
S_{n+1}+u_{n} S_{n-1}(x)=x S_{n}(x),
$$

where the recurrence coefficients are

$$
u_{n}=\left(1+a_{n-1}\right)\left(1-a_{n-2}\right), \quad n=1,2, \ldots
$$

In (5.13) it is assumed that $a_{-1}=-1$ (this is a standard convention in the theory of polynomials orthogonal on the unit circle), so $u_{1}=2\left(1+a_{0}\right)$.

If the polynomials $\tilde{P}_{n}(z)$ are orthogonal on the unit circle

$$
\int_{0}^{2 \pi} \tilde{P}_{n}\left(e^{i \theta}\right) \tilde{P}_{m}^{*}\left(e^{-i \theta}\right) \rho(\theta) d \theta=0, \quad m \neq n
$$

with the weight function $\rho(\theta)$ then polynomials $S_{n}(x)$ are orthogonal on the symmetric interval $[-2,2]$

$$
\int_{-2}^{2} S_{n}(x) S_{m}(x) w(x) d x=h_{n} \delta_{n m}
$$


with the weight function $[4,26]$

$$
w(x)=\frac{\rho(\theta)}{\sin (\theta / 2)},
$$

where $x=2 \cos (\theta / 2)$.

In our case it is elementary verified that

$$
u_{n}=\frac{(n+1 / 2)^{2}}{n(n+1)}, \quad n=1,2, \ldots
$$

These recurrence coefficients correspond to well known associated Legendre polynomials studied, e.g. in [1]. Recall that generic associated Legendre polynomials are symmetric OP satisfying the recurrence relation (5.12) with the recurrence coefficients

$$
u_{n}=\frac{(n+\nu)^{2}}{(n+\nu)^{2}-1 / 4}
$$

with arbitrary nonnegative parameter $\nu$. The ordinary Legendre polynomials correspond to $\nu=0$. In our case we have $\nu=1 / 2$.

Consider the weight function for these polynomials. From (5.16) and (5.6) we derive

$$
w(x)=\frac{1}{2\left|K\left(e^{-i \theta / 2}\right)\right|^{2}} .
$$

We can simplify this formula if one exploits the relations for $K(z)$ where $|z|=1$. Namely, one has $[17]$

$$
K\left(e^{ \pm i \phi}\right)=\frac{1}{2} e^{\mp i \phi / 2}(K(\cos (\phi / 2)) \pm i K(\sin (\phi / 2))), \quad-\pi<\phi \leq \pi,
$$

whence we have

$$
w(x)=\frac{2}{K^{2}(\cos (\theta / 4))+K^{2}(\sin (\theta / 4))} .
$$

Taking into account that $x=2 \cos (\theta / 2)$ we finally arrive at the formula

$$
w(x)=\frac{2}{K^{2}(\sqrt{1 / 2+x / 4})+K^{2}(\sqrt{1 / 2-x / 4})}, \quad-2 \leq x \leq 2 .
$$

Note that the function $w(x)$ is even $w(-x)=w(x)$ as should be for symmetric polynomials. It has the only maximum at $x=0: w(0)=1 / K^{2}(\sqrt{1 / 2})=\frac{16 \pi}{\Gamma^{4}(1 / 4)}$. Near the endpoints of the interval $[-2,2]$ the weight function $w(x)$ tends to zero rapidly.

The weight function for generic associated Legendre polynomials (with arbitrary $\nu$ ) was found in [1]. Our formula (5.21) can be obtained from the results of [1] by putting $\nu=1 / 2$. Note, that Pollaczek studied [18] more general orthogonal polynomials containing 4 parameters. The associated Legendre polynomials (as well as the associated ultraspherical polynomials) are contained in the Pollaczek polynomial family as a special case.

Similar polynomials were studied also in [19] where the author in fact rediscovered the Hermite approach (as well Hermite's continued fraction (1.9)) to elliptic integrals. He considered integrals $J_{n}(k)$ as moments for some "elliptic" orthogonal polynomials having $w(x)=$ $1 / \sqrt{\left(1-x^{2}\right)\left(1-k^{2} x^{2}\right)}$ as an orthogonality weight on the interval $[-1,1]$. As a by-product the author of [19] introduced polynomials which are similar to the Hermite polynomials $A_{n}(z)$, 
$B_{n}(z)$. He then related them with the associated Legendre polynomials in a similar way. It is interesting to note that Hermite himself already introduced such polynomials in [10, 11]. Hermite also established differential properties of these polynomials anticipating results of Rees [19].

Consider relations between LBP of special type and orthogonal polynomials on an interval in details.

We have $\tilde{P}_{n}(z)=\left(P_{n+1}(z)-U_{n} P_{n}(z)\right) /(z-1)$. Substituting this formula to (5.11) and using the inversion property $z^{n} P_{n}(1 / z)=P_{n}(z)$ of our LBP $P_{n}(z)$ we immediately obtain a very simple relation

$$
S_{n}(x)=z^{-n / 2} P_{n}(z)
$$

We can see this also using recurrence relation for the LBP

$$
P_{n+1}(z)+d_{n} P_{n}(z)=z\left(P_{n}(z)+b_{n} P_{n-1}(z)\right)
$$

Assume (as in our case) that $d_{n}=-1, n=0,1, \ldots$ Then $P_{n}(0)=1$ for all $n=0,1,2, \ldots$ and from (2.11) we obtain that the reciprocal polynomials coincide with the initial ones:

$$
z^{n} P_{n}(1 / z)=P_{n}(z)
$$

Conversely, assume that some LBP are reciprocal (5.24). Then from (2.11) it follows that either $d_{n}=1, n=0,1, \ldots$ or $d_{n}=-1, n=0,1, \ldots$ But we have obviously $P_{n}(0)=1$ which leads to the only possibility $d_{n}=-1, n=0,1,2, \ldots$ Thus condition $d_{n}=-1$ for all $n$ is necessary and sufficient for LBP to be reciprocal invariant (5.24). In this case polynomials

$$
S_{n}(x)=z^{-n / 2} P_{n}(z)
$$

are obviously monic polynomials in $x=z^{1 / 2}+z^{-1 / 2}$. From recurrence relation (5.23) with $d_{n}=-1$ we obtain recurrence relation for polynomials $S_{n}(x)$

$$
S_{n+1}(x)+u_{n} S_{n-1}(x)=x S_{n}(x),
$$

where $u_{n}=-b_{n}$. Thus we arrived at the same symmetric polynomials on the interval as in the case of polynomials orthogonal on the unit circle. Note that for the LBP $P_{n}(z)$ with the property (5.24) we have $c_{1}=d_{0}=-1$ and $c_{n}^{*}=c_{n}$ thus from (2.12) we obtain

$$
c_{1-n}=-c_{n}
$$

for all $n=0, \pm 1, \pm 2, \ldots$. Perform now the Christoffel transform with $\mu=1$

$$
\tilde{P}_{n}(z)=\frac{P_{n+1}(z)-U_{n} P_{n}(z)}{z-1}
$$

From (2.19) and (5.27) we obtain that transformed moments are symmetric $\tilde{c}_{-n}=c_{n}$. This means that polynomials $\tilde{P}_{n}(z)$ will satisfy the recurrence relation (5.7) with reflection parameters $a_{n}$ given by $a_{n}=1-U_{n+1}=1-P_{n+2}(1) / P_{n+1}(1)$. Formula (5.11) now is equivalent to formula (5.25). Thus starting from arbitrary LBP with the property $d_{n}=-1$ we can arrive at the same symmetric OP on the interval $S_{n}(x)$ as for the case of polynomials orthogonal on the unit circle. From another point of view these relations are discussed also in [2]. 


\section{Geronimus transform. Laurent biorthogonal polynomials with a concentrated mass added to the measure}

In the previous section we showed that the Christoffel transformation of Hermite's elliptic LBP gives polynomials orthogonal on the unit circle with explicit reflection coefficients (5.10) and the weight function given by (5.6). In this section we consider another spectral transformation of Hermite's elliptic LBP which is called the Geronimus transform (GT). This transform was introduced in [27] and is similar to well known Geronimus transform for the ordinary orthogonal polynomials [20, 25]. Recall thatGT for LBP is defined as [27]

$$
\tilde{P}_{n}(z)=V_{n} P_{n}(z)+z\left(1-V_{n}\right) P_{n-1}(z),
$$

where

$$
V_{0}=1, \quad V_{n}=\frac{\mu}{\mu-\phi_{n} / \phi_{n-1}}, \quad n=1,2, \ldots
$$

In (6.2) $\mu$ is an arbitrary parameter and $\phi_{n}$ is an arbitrary solution of the recurrence relation

$$
\phi_{n+1}+d_{n} \phi_{n}=\mu\left(\phi_{n}+b_{n} \phi_{n-1}\right) .
$$

Note that (6.3) is the same recurrence relation that (2.2) for LBP. Hence its the general solution (up to a common factor) can be presented in the form

$$
\phi_{n}=P_{n}(\mu)+\chi P_{n-1}^{(1)}(\mu) .
$$

It is easy to verify that the polynomials $\tilde{P}_{n}(z)$ are again LBP satisfying recurrence relation

$$
\tilde{P}_{n+1}(z)+\tilde{d}_{n} \tilde{P}_{n}(z)=z\left(\tilde{P}_{n}(z)+\tilde{b}_{n} \tilde{P}_{n-1}(z)\right),
$$

where the recurrence coefficients are

$$
\tilde{b}_{1}=\chi\left(V_{1}-1\right), \quad \tilde{b}_{n}=b_{n-1} \frac{1-V_{n}}{1-V_{n-1}}, \quad \tilde{d}_{n}=d_{n} \frac{V_{n+1}}{V_{n}} .
$$

It can be shown that $\mathrm{CT}$ and GT are reciprocal to one another [27]. This observation allows one to obtain explicit biorthogonality relation for polynomials $\tilde{P}_{n}(z)$ starting from that for polynomials $P_{n}(z)$. Namely, the pair of the Stieltjes functions $F_{+}(z), F_{-}(z)$ is transformed as [27]

$$
\tilde{F}_{+}(z)=\frac{\nu F_{+}(z)+\mu+\nu}{z-\mu}, \quad F_{-}(z)=\frac{\nu F_{-}(z)-\mu-\nu}{z-\mu},
$$

where

$$
\nu=\frac{\mu \chi}{c_{1}-\chi}=\frac{\mu \chi}{d_{0}-\chi} .
$$

Assume that $|\mu| \leq 1$. Choose the contour $C$ as the unit circle (if $|\mu|=1$ we choose $C$ as the unit circle with a small deformation near $z=\mu$ in order to include the point $z=\mu$ inside the contour $C$ ). Then the weight function for new polynomials $\tilde{P}_{n}(z)$ is defined as in $(3.2)$, i.e.

$$
\tilde{w}(z)=\frac{\tilde{F}_{+}(z)-\tilde{F}_{-}(z)}{2 \pi i z}=\frac{w(z)}{z-\mu}+\frac{2(\mu+\nu)}{2 \pi i z(z-\mu)} .
$$

The second term in (6.8) will give a concentrated mass at the point $z=\mu$ added to a "regular" part presented by the first term in (6.8). 
Assume that $\mu=1$, as in our case. Then we have biorthogonality relation

$$
\int_{0}^{2 \pi} \tilde{\rho}(\theta) \tilde{P}_{n}\left(e^{i \theta}\right) \hat{\tilde{P}}_{n}\left(e^{-i \theta}\right) d \theta+M \tilde{P}_{n}(1) \hat{\tilde{P}}_{n}(1)=0, \quad n \neq m
$$

where

$$
\tilde{\rho}(\theta)=\frac{\rho(\theta)}{e^{i \theta}-1}
$$

is the "regular" of the weight function on the unit circle and the last term (6.10) describes the concentrated mass

$$
M=\frac{\nu+1}{2 \pi}
$$

inserted at the point $z=1$.

In our case we have $d_{n}=-1, n=0,1, \ldots$ hence $\nu=-\chi /(\chi+1)$ and

$$
M=\frac{1}{2 \pi(1+\chi)}
$$

For the recurrence coefficients we have explicit formulas (6.6) where we need first to calculate the coefficients $V_{n}$. We see that

$$
\phi_{n}=P_{n}(1)+\chi P_{n-1}^{(1)}(1) .
$$

But the values $P_{n}(1)$ and $P_{n-1}^{(1)}(1)$ were already calculated (see (4.8), (4.12)). We thus have

$$
\phi_{n}=\frac{(3 / 2)_{n}}{n !}\left(G_{n}+2 \chi\left(G_{n}-1\right)\right)
$$

Now all coefficients $V_{n}, \tilde{b}_{n}, \tilde{d}_{n}$ are calculated explicitly.

We thus constructed a nontrivial example of the Laurent biorthogonal polynomials with explicit both recurrence coefficients and the measure. The weight function for these polynomials has a concentrated mass on the unit circle. Note that in contrast to the Christoffel transformed Hermite's polynomials the polynomials $\tilde{P}_{n}(z)$ constructed in this section are not polynomials of the Szegö type. This means, in particular, that the biorthogonal partners $\hat{\tilde{P}}_{n}(z)$ do not coincide with $\tilde{P}_{n}(z)$.

\section{Associated families of the Laurent biorthogonal polynomials}

Return to the sequence $J_{n}(x, k)$ of incomplete elliptic integrals defined by (1.2). They satisfy three-term recurrence relation (1.3). Repeating previous considerations we can express $J_{n}(x ; k)$ for any $n=1,2, \ldots$ in terms of $J_{j}(x ; k)$ and $J_{j+1}(x ; k)$ for some fixed nonnegative integer $j$ (in (1.4) the case $j=0$ is chosen):

$$
J_{n+1}(x ; k)=Q(x ; n, j) \sqrt{\left(1-x^{2}\right)\left(1-k^{2} x^{2}\right)}+A_{n-j}^{(j)} J_{j+1}(x ; k)-B_{n-j}^{(j)} J_{j}(x ; k)
$$

with some coefficients $A_{n}^{(j)}, B_{n}^{(j)}$.

We first note that there is an explicit expression of complete elliptic integrals $J_{n}(k)$ in terms of the Gauss hypergeometric function

$$
J_{n}(k)=\frac{k^{2 n} \pi(1 / 2)_{n}}{2 n !}{ }_{2} F_{1}\left(1 / 2,1 / 2+n ; 1+n ; k^{2}\right)
$$


(relation (7.2) can be easily verified by direct integration).

Repeating similar considerations that were already exploited in the two first sections we can show that $A_{n}^{(j)}(z)$ and $B_{n}^{(j)}$ are determined by the recurrence relations

$$
\begin{aligned}
& (n+3 / 2+j) A_{n+1}^{(j)}=(n+j+1)(z+1) A_{n}^{(j)}-z(n+j+1 / 2) A_{n-1}^{(j)}, \\
& (n+3 / 2+j) B_{n+1}^{(j)}=(n+j+1)(z+1) B_{n}^{(j)}-z(n+j+1 / 2) B_{n-1}^{(j)}
\end{aligned}
$$

with the same initial conditions as (1.6). Hence, $A_{n}^{(j)}(z)$ polynomials of $n$-th degree in $z=k^{2}$ and $B_{n}^{(j)}(z)$ is a polynomial of degree $n-1$ multiplied by $z$.

Introduce also the function

$$
F(z ; j)=\frac{J_{j+1}(k)}{J_{j}(k)},
$$

where as usual we put $k^{2}=z$.

Then we will have the property

$$
F(z ; j)-\frac{B_{n}^{(j)}(z)}{A_{n}^{(j)}(z)}=O\left(z^{n+1}\right) .
$$

In the same way it is possible to derive the generating functions for polynomials $A_{n}^{(j)}(z)$, $B_{n}^{(j)}(z)$ :

$$
\begin{aligned}
& (2 j+1) \frac{k^{-2 j} J_{j}(x ; k)}{\sqrt{\left(1-x^{2}\right)\left(1-k^{2} x^{2}\right)}}=\sum_{n=j}^{\infty} A_{n-j}^{(j)}(z) x^{2 n+1}, \\
& (2 j+1) \frac{k^{-2 j} J_{j+1}(x ; k)}{\sqrt{\left(1-x^{2}\right)\left(1-k^{2} x^{2}\right)}}=\sum_{n=j}^{\infty} B_{n-j}^{(j)}(z) x^{2 n+1} .
\end{aligned}
$$

Using (4.6) we arrive at the explicit representation for polynomials $A_{n}^{(j)}(z)$ in terms of the Legendre polynomials $Y_{n}(q)$ :

$$
A_{n}^{(j)}(z)=(2 j+1) k^{n} \sum_{s=0}^{n} \frac{Y_{s}(q) Y_{n-s}(q)}{2 s+2 j+1},
$$

where $z=k^{2}, q=(k+1 / k) / 2$.

Now we introduce the monic LBP $P_{n}^{(j)}(z)=A_{n}^{(j)}(z) / \xi_{n}$ where $\xi_{n}=(j+1)_{n} /(j+3 / 2)_{n}$. They satisfy the recurrence relation of type (2.2) with

$$
d_{n}=-1, \quad b_{n}=-\frac{(n+j+1 / 2)^{2}}{(n+j)(n+j+1)} .
$$

Thus we have $j$-associated polynomials with respect to the Hermite elliptic LBP, i.e. we should replace $n \rightarrow n+j$ in formulas for recurrence coefficients.

Due to the property $d_{n}=-1$ the associated polynomials $P_{n}^{(j)}$ again possess the invariance property $z^{n} P_{n}^{(j)}(1 / z)=P_{n}^{(j)}(z)$. We thus can construct polynomials orthogonal on the unit circle using formula (5.28). In order to get explicit expression for reflection parameters $a_{n}^{(j)}$ we need the value $P_{n}^{(j)}(1)$. But this can easily be obtained from (7.7):

$$
A_{n}^{(j)}(1)=G_{n}(j)
$$


where we introduced the function

$$
G_{n}(j)=(2 j+1) \sum_{s=0}^{n} \frac{1}{2 s+2 j+1} .
$$

(Up to a common factor $G_{n}(j)$ is a sum of $n+1$ succeeding inverse odd numbers starting from $1 /(2 j+1)$; for $j=0$ it coincides with $G_{n}$.) Thus

$$
U_{n}=\frac{P_{n+1}^{(j)}(1)}{P_{n}^{(j)}(1)}=\frac{n+3 / 2+j}{n+j+1} \frac{G_{n}(j)}{G_{n+1}(j)}
$$

and

$$
a_{n}^{(j)}=1-U_{n+1}
$$

Corresponding symmetric polynomial $S_{n}^{(j)}(x)$ on the interval satisfy recurrence relation (5.26) with

$$
u_{n}=\frac{(n+j+1 / 2)^{2}}{(n+j)(n+j+1)}
$$

so they coincide with the associated Legendre polynomials considered in [1]. Indeed, from formula (5.18) we see that the "shift" parameter $\nu=j+1 / 2$ where $j=0,1,2, \ldots$

It is interesting to note that recurrence relations for polynomials $P_{n}^{(j)}$ can be presented in such a form that the all coefficients are linear in $n$ - see e.g. (7.3). In [6] we considered a family of such LBP connected with so-called generalized eigenvalue problem on $s u(1,1)$ Lie algebra. In our case, however, corresponding representations of $s u(1,1)$ will not be unitary, in contrast to [6]. This leads to an interesting open problem how to describe associated classical LBP in terms of non-unitary representations of $s u(1,1)$ algebra. Note that generic orthogonal polynomials with linear recurrence coefficients in $n$ were studied in details by Pollaczek [18] who derived explicit expression for them and found the weight function as well.

Note finally that LBP with recurrence coefficients (7.8) belong to a family of so-called "associated Jacobi Laurent polynomials" introduced and studied by Hendriksen [8, 9]. Nevertheless, in our case the parameters of the associated LBP belong to the exceptional class which was not considered in $[8,9]$. This means that in some formulas in $[8,9]$ the bottom parameter in the Gauss hypergeometric function ${ }_{2} F_{1}(z)$ takes negative integer values. In this case formulas obtained by Hendriksen should be rederived in a different form. In particular, a simple explicit expression of the power coefficients (in terms of the hypergeometric function ${ }_{4} F_{3}(1)$ ) for associated LBP obtained in [8] seems not to be valid in our case. Instead, we obtained explicit expressions like (7.7).

\section{Laurent biorthogonal polynomials connected with the Stieltjes-Carlitz elliptic polynomials}

Return to formula (1.1). If one denotes

$$
r_{n}(p)=\int_{0}^{\infty} k^{2 n} s n^{2 n}(t) e^{-p t} d t
$$

then we obtain the recurrence relation

$$
p^{2} r_{n}=2 n(2 n+1) r_{n+1}-4\left(1+k^{2}\right) n^{2} r_{n}+2 n(2 n-1) r_{n-1} .
$$


Again it is seen that for every $n>0$ one can present

$$
r_{n+1}=A_{n} r_{1}-B_{n} r_{0},
$$

where obviously $r_{0}=\int_{0}^{\infty} e^{-p t} d t=p^{-1}$ and $A_{n}, B_{n}$ satisfy the same recurrence relations as $r_{n+1}$ e.g.

$$
p^{2} A_{n}=2(n+1)(2 n+1) k^{2} A_{n-1}-4\left(1+k^{2}\right)(n+1)^{2} A_{n}+2(n+1)(2 n+3) A_{n+1}
$$

with initial conditions $A_{0}=1, A_{-1}=0, B_{0}=0, B_{-1}=-1$. Now it is seen that $A_{n}$ are polynomials of degree $n$ in both variables $p^{2}$ and $k^{2}$. If $p=0$ then $A_{n}$ become polynomials in $k^{2}$ introduced by Hermite.

Coefficients $A_{n}$ considered as polynomials in $p^{2}$ become orthogonal polynomials because they satisfy three-term recurrence relation typical for orthogonal polynomials. Orthogonal polynomials of such type (and several related ones) where introduced and studied by Carlitz. He exploited some explicit continued fractions found by Stieltjes. These continued fractions are connected with elliptic functions (for details see, e.g. [13]). Today these orthogonal polynomials are known by Stieltjes-Carlitz elliptic polynomials [16, 3, 13]. Note that the Stieltjes continued fraction is obtained from (8.2) by the same way as Hermite obtained his continued fraction (1.9) for a ratio of two elliptic integrals. Consider now polynomials $A_{n}$ as LBP with respect to the argument $z=k^{2}$. Passing from $A_{n}$ to monic polynomials $P_{n}(z)$ (by the same way as for the case $p=0$ ) we arrive at the recurrence relation (2.2) with

$$
d_{n}=-1-\frac{p^{2}}{4(n+1)^{2}}, \quad b_{n}=-\frac{(n+1 / 2)^{2}}{n(n+1)} .
$$

We see that the recurrence coefficient $b_{n}$ is the same as for the Hermite LBP, but the coefficient $d_{n}$ now depends on $n$. This means that polynomials $P_{n}(z)$ do not possess symmetric property like (2.13).

In contrast to the case $p=0$ the polynomials $P_{n}(z)$ have more complicated properties. For example, the reciprocal polynomials $P_{n}^{*}(z)$ defined by $(2.10)$ do not belong to the same class, their recurrence coefficients appear to be

$$
d_{n}^{*}=-\frac{1}{1+\frac{p^{2}}{4(n+1)^{2}}}, \quad b_{n}^{*}=-\frac{(n+1)(n+2)^{2}(n+1 / 2)^{2}}{\left((n+2)^{2}+p^{2} / 4\right)\left((n+1)^{2}+p^{2} / 4\right)} .
$$

The biorthogonal partners $\hat{P}_{n}(z)$ defined by $(2.15)$ have the recurrence coefficients

$$
\begin{aligned}
& \hat{d}_{n}=-\frac{n(n+1)\left(p^{2}(n+1)-n-2\right)}{\left(n\left(p^{2}-1\right)-1\right)\left((n+2)^{2}+p^{2} / 4\right)} \\
& \hat{b}_{n}=-\frac{(n+1 / 2)^{2}(n+1)^{2}\left(p^{2}(n+1)-n-2\right)}{\left(n\left(p^{2}-1\right)-1\right)\left((n+1)^{2}+p^{2} / 4\right)\left((n+2)^{2}+p^{2} / 4\right)} .
\end{aligned}
$$

Hence biorthogonal partners $\hat{P}_{n}(z)$ also do not belong to the same class that initial LBP $P_{n}(z)$.

For the ordinary Stieltjes-Carlitz elliptic orthogonal polynomials the orthogonality measure can be found explicitly: it is purely discrete one (see [3, 13]). The problem of finding the orthogonality measure for the corresponding LBP seems to be much more complicated.

\section{Acknowledgments}

The authors thank to the referees for their remarks leading to improvement of the text. A.Zh. thanks Centre de Recherches Mathématiques of the Université de Montréal for hospitality. 


\section{References}

[1] Barrucand P., Dickinson D., On the associated Legendre polynomials, in Orthogonal Expansions and Their Continued Analogues, Editor D.T. Haimo, Southern Illinois Press, 1968, 43-50.

[2] Bracciali C.F., da Silva A.P., Sri Ranga A., Szegö polynomials: some relations to $L$-orthogonal and orthogonal polynomials, J. Comput. Appl. Math. 153 (2003), 79-88.

[3] Chihara T., An introduction to orthogonal polynomials, Gordon and Breach, 1978.

[4] Delsarte P., Genin Y., The split Levinson algorithm, IEEE Trans. Acoust. Speech Signal Process 34 (1986), 470-478.

[5] Geronimus Ya.L., Polynomials orthogonal on a circle and their applications, in Amer. Math. Soc. Transl. Ser. 1, Vol. 3, Amer. Math. Soc., Providence, 1962, 1-78.

[6] Grünbaum F.A., Vinet L., Zhedanov A., Linear operator pencils on Lie algebras and Laurent biorthogonal polynomials, J. Phys. A: Math. Gen. 37 (2004), 7711-7725.

[7] Hendriksen E., van Rossum H., Orthogonal Laurent polynomials, Indag. Math. (Ser. A) 48 (1986), 17-36.

[8] Hendriksen E., Associated Jacobi-Laurent polynomials, J. Comput. Appl. Math. 32 (1990), 125-141.

[9] Hendriksen E., A weight function for the associated Jacobi-Laurent polynomials, J. Comput. Appl. Math. 33 (1990), 171-180.

[10] Hermite C., Sur la développement en série des integrales elliptiques de premiere et de seconde espece, Annali di Matematica II (1868), 2 ser., 97-97.

[11] Hermite C., Oeuvres, Tome II, Paris, 1908, 486-488.

[12] Hermite C., Cours d'analyse de la Faculté des Sciences, Editor Andoyer, Hermann, Paris, 1882 (Lithographed notes).

[13] Ismail M.E.H., Masson D., Some continued fractions related to elliptic functions, Contemp. Math. 236 (1999), 149-166.

[14] Jones W.B., Thron W.J., Survey of continued fraction methods of solving moment problems, in Analytic Theory of Continued Fractions, Lecture Notes in Math., Vol. 932, Springer, Berlin - Heidelberg - New York, 1981.

[15] Koekoek R., Swarttouw R.F., The Askey scheme of hypergeometric orthogonal polynomials and its $q$ analogue, Report 94-05, Delft University of Technology, Faculty of Technical Mathematics and Informatics, 1994.

[16] Lomont J.S., Brillhart J., Elliptic polynomials, Chapman \& Hall/CRC, Boca Raton, FL, 2001.

[17] Magnus W., Oberhettinger F., Formeln und Sätze fur die speziellen Functionen der mathematischen Physik, Springer, Berlin, 1948.

[18] Pollaczek F., Sur une famille de polynômes orthogonaux à quatre paramitres, C. R. Acad. Sci. Paris 230 (1950), 2254-2256.

[19] Rees C.J., Elliptic orthogonal polynomials, Duke Math. J. 12 (1945), 173-187.

[20] Spiridonov V., Vinet L., Zhedanov A., Spectral transformations, self-similar reductions and orthogonal polynomials, J. Phys. A: Math. Gen. 30 (1997), 7621-7637.

[21] Szegö G., Orthogonal polynomials, AMS, 1959.

[22] Vinet L., Zhedanov A., An integrable chain and bi-orthogonal polynomials, Lett. Math. Phys. 46 (1998), 233-245.

[23] Vinet L., Zhedanov A., Spectral transformations of the Laurent biorthogonal polynomials. I. $q$-Appel polynomials, J. Comput. Appl. Math. 131 (2001), 253-266.

[24] Whittacker E.T., Watson G.N., A course of modern analysis, 4th ed., Cambridge University Press, 1927.

[25] Zhedanov A., Rational spectral transformations and orthogonal polynomials, J. Comput. Appl. Math. 85 (1997), 67-86.

[26] Zhedanov A., On some classes of polynomials orthogonal on arcs of the unit circle connected with symmetric orthogonal polynomials on an interval, J. Approx. Theory 94 (1998), 73-106.

[27] Zhedanov A., The "classical" Laurent biorthogonal polynomials, J. Comput. Appl. Math. 98 (1998), 121147 . 